\title{
Structural Studies on MtRecA-Nucleotide Complexes: Insights Into DNA and Nucleotide Binding and the Structural Signature of NTP Recognition
}

\author{
S. Datta, ${ }^{1}$ N. Ganesh, ${ }^{2}$ Nagasuma R. Chandra, ${ }^{3 *}$ K. Muniyappa, ${ }^{2}$ and M. Vijayan ${ }^{1 *}$ \\ ${ }^{1}$ Molecular Biophysics Unit, Indian Institute of Science, Bangalore, India \\ ${ }^{2}$ Department of Biochemistry, Indian Institute of Science, Bangalore, India \\ ${ }^{3}$ Bioinformatics Centre, Indian Institute of Science, Bangalore, India
}

ABSTRACT RecA protein plays a crucial role in homologous recombination and repair of DNA. Central to all activities of RecA is its binding to $\mathrm{Mg}^{+2}$-ATP. The active form of the protein is a helical nucleoprotein filament containing the nucleotide cofactor and single-stranded DNA. The stability and structure of the helical nucleoprotein filament formed by RecA are modulated by nucleotide cofactors. Here we report crystal structures of a MtRecAADP complex, complexes with ATP $\gamma \mathrm{S}$ in the presence and absence of magnesium as well as a complex with dATP and $\mathrm{Mg}^{+2}$. Comparison with the recently solved crystal structures of the apo form as well as a complex with $\mathrm{ADP}^{-\mathrm{AlF}_{4}}$ confirms an expansion of the P-loop region in MtRecA, compared to its homologue in Escherichia coli, correlating with the reduced affinity of MtRecA for ATP. The ligand bound structures reveal subtle variations in nucleotide conformations among different nucleotides that serve in maintaining the network of interactions crucial for nucleotide binding. The nucleotide binding site itself, however, remains relatively unchanged. The analysis also reveals that $\mathrm{ATP} \gamma \mathrm{S}$ rather

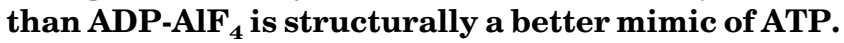
From among the complexed structures, a definition for the two DNA-binding loops L1 and L2 has clearly emerged for the first time and provides a basis to understand DNA binding by RecA. The structural information obtained from these complexes correlates well with the extensive biochemical data on mutants available in the literature, contributing to an understanding of the role of individual residues in the nucleotide binding pocket, at the molecular level. Modeling studies on the mutants again point to the relative rigidity of the nucleotide binding site. Comparison with other NTP binding proteins reveals many commonalties in modes of binding by diverse members in the structural family, contributing to our understanding of the structural signature of NTP recognition. Proteins 2003;50:474-485.

$\odot 2003$ Wiley-Liss, Inc.

\section{INTRODUCTION}

RecA protein, a well-conserved multi-functional protein, promotes the central steps in genetic recombination and DNA repair and is also closely associated with the induc- tion of SOS response to DNA damage. ${ }^{1}$ A fundamental pre-requisite for all activities of RecA protein is its binding the nucleotide cofactor $\mathrm{Mg}^{+2}$-ATP. The biochemically active conformation of RecA, triggered by the nucleotide binding, is a helical nucleoprotein filament comprising RecA-ATP-ssDNA. The structure and stability of the helical nucleoprotein filament formed by RecA are modulated by nucleotide cofactors. ${ }^{2}$ While known to be crucial, the exact role of ATP in RecA-mediated strand exchange has yet to be unambiguously established. According to one hypothesis, the energy derived from ATP hydrolysis is strongly coupled with DNA strand exchange while another hypothesis implies that ATP is mainly an allosteric cofactor inducing conformational changes in the protein, thus enhancing its DNA binding ability. ${ }^{3,4}$ A recent report suggests that the effect of ATP on DNA affinity is a result of an increase in cooperative assembly of the protein itself rather than an increase in the intrinsic affinity for DNA. ${ }^{5,6}$

A prerequisite for understanding the role of this vital co-factor in the mechanism of RecA function is a thorough knowledge of RecA-nucleotide interactions. We have recently solved the crystal structures of RecA protein from Mycobacterium tuberculosis (MtRecA) and its complex with $\mathrm{ADP}-\mathrm{AlF}_{4}{ }^{7}$ Here we report structures of the complexes of MtRecA with different nucleotides, in which the two DNA binding loops too are clearly characterized. These crystal structures represent the first RecA-nucleotide complexes to be clearly defined from any species and they provide detailed information about RecA-nucleotide interactions in general. They also represent the first RecA structures in which the two crucial loops have been elucidated, thus providing a framework for understanding the DNA binding by RecA. In addition to determining the crystal structures, molecular models were constructed for 21 site-directed mutants of the P-loop and analyzed in the context of their biochemical properties, in an effort to clearly define the role of each residue of the P-loop. A comparison with other P-loop-containing structures was

Grant sponsor: Department of Biotechnology, Government of India.

*Correspondence to: Prof. M. Vijayan, Molecular Biophysics Unit, or Dr. N.R. Chandra, Bioinformatics Centre, Indian Institute of Science, Bangalore 560 012, India. E-mail: mv@mbu.iisc.ernet.in

Received 25 June 2002; Accepted 25 September 2002 
also carried out specifically to study commonalities and differences in modes of nucleotide binding and more generally to understand fold-function relationship in the structural family.

\section{METHODS \\ Crystallisation and Data Collection}

MtRecA was purified as described earlier. ${ }^{8} \mathrm{ADP}$ and ATP $\gamma$ S were obtained from Amersham-Pharmacia. Native MtRecA crystals were grown from a hanging drop of 10 $\mathrm{mg} / \mathrm{ml}$ protein in $100 \mathrm{mM}$ tris-acetate buffer, $\mathrm{pH}$ 7, containing $100 \mathrm{mM} \mathrm{NaCl}$ and 6\% PEG 4000 equilibrated against $20 \%$ PEG 4000 in the same buffer. Crystals of the MtRecAADP, MtRecA-dATP-Mg ${ }^{+2}$, and MtRecA-ATP $\gamma \mathrm{S}-\mathrm{Mg}^{+2}$ complexes were grown by co-crystallization under similar conditions but in the presence of $2 \mathrm{mM}$ of the appropriate ligand. Crystals of MtRecA-ATP $\gamma \mathrm{S}$ (in the absence of magnesium) were obtained by soaking native crystals in the mother liquor containing $2 \mathrm{mM} \mathrm{ATP} \gamma \mathrm{S}$ for one week. $\mathrm{X}$-ray diffraction data from all complexes were collected at room temperature using a 300-mm MAR imaging plate mounted on a Rigaku RU200 X-ray generator. In all cases, crystal-to-plate distance was maintained at $110 \mathrm{~mm}$. The complexes diffracted to a resolution of 3.25-3.1 $\AA$. All data were processed using DENZO and SCALEPACK program suites $^{9}$ in an identical manner. The data-collection statistics are given in Table I.

\section{Structure Refinement}

The protein atoms of native MtRecA structure ${ }^{7,10}$ (PDB: 1G19) were used as the starting model. The structures were refined in an identical manner using CNS. ${ }^{11}$ Initially, the structures were subjected to rigid body refinement, treating the three domains (N:1-30,M:31-269,C:270-329) of the protein as rigid groups. This was followed by cycles of positional refinement and simulated annealing. Both $2 \mathrm{~F}_{\mathrm{o}}-\mathrm{F}_{\mathrm{c}}$ and $\mathrm{F}_{\mathrm{o}}-\mathrm{F}_{\mathrm{c}}$ electron density maps were calculated at this stage. The $\mathrm{F}_{\mathrm{o}}-\mathrm{F}_{\mathrm{c}}$ difference Fourier maps permitted an initial unambiguous positioning of the nucleotides in all the complexes. In view of the low observations to parameter ratio, grouped-B factor refinements were carried out instead of the individual atomic B factor refinements. To remove the phase bias during refinement, a systematic omit map ${ }^{12}$ was computed in each case, which in essence is a summation of eight omit maps in each of which an eighth of the asymmetric unit was systematically omitted. Such an omit map has been shown to have the advantage of providing an image of the entire structure that is formally independent of the input model. For each of the four structures, simulated annealed omit maps were also calculated, where a sphere of $6 \AA$ around the ligand was omitted. These omit maps were used along with the $2 \mathrm{~F}_{\mathrm{O}}-\mathrm{F}_{\mathrm{C}}$ and $\mathrm{F}_{\mathrm{O}}-\mathrm{F}_{\mathrm{C}}$ maps for model building using FRODO ${ }^{13}$ into the electron density. At this stage, the electron density corresponding to the DNA binding loop L1 in the MtRecA-ATP $\gamma \mathrm{S}-\mathrm{Mg}^{+2}$ structure became interpretable and allowed unambiguous building of the whole loop into the density. Similarly, residues in the DNA binding loop L2 in MtRecA-ATP $\gamma \mathrm{S}$ could be built into the electron density map. The geometric parameters for ADP, ATP $\gamma \mathrm{S}$, and dATP used in the refinement were obtained from the HICCUP database. ${ }^{14} \mathrm{~A}$ few water molecules were built into the density, where the peaks were visible at contours of at least $2.5 \sigma$ in $\mathrm{F}_{\mathrm{o}}-\mathrm{F}_{\mathrm{c}}$ and $0.8 \sigma$ in $2 \mathrm{~F}_{\mathrm{o}}-\mathrm{F}_{\mathrm{c}}$ electron density maps. Cycles of positional refinement and correction of the model using Fourier maps were repeated. Refinement parameters for all the structures are given in Table I. Positional coordinates and structure factors of the four complexes are deposited in the Protein Data Bank. The PDB codes are IMO3, IMO4, IMO5, and IMO6.

\section{Geometric Analysis and Modeling}

The stereochemical quality of the structures was validated using PROCHECK. ${ }^{15}$ Information on the occurrence of residues in different regions of the Ramachandran plot is given in Table I. Surface areas were calculated using Connolly algorithm ${ }^{16}$ employing a probe radius of $1.2 \AA$. Superposition of structures was carried out using ALIGN. ${ }^{17}$ A distance of less than $3.6 \AA$ between donor and acceptor atoms and an angle greater than $90^{\circ}$ at the hydrogen atom were used as criterion for determining hydrogen bonds. Hydrogens were fixed geometrically using the CNS program suite. Model building of site-directed mutants and energy minimizations were carried out using the DISCOVER program suite interfaced with Insight-II (Accelrys Inc.), employing standard parameters.

\section{RESULTS AND DISCUSSION \\ Overall Structure}

The structure of RecA protein consists of a major central domain (M), flanked by two smaller domains at the amino and carboxy termini ( $\mathrm{N}$ and $\mathrm{C}$, respectively) as illustrated in Figure 1. The 30 residue $\mathrm{N}$ domain consisting of a long $\alpha$-helix followed by an extended region, clearly projects out of the $\mathrm{M}$ domain and plays a major role in stabilizing aggregation of the protein molecules into filaments. The M domain belonging to the SCOP classification ${ }^{18}$ of "P-loop containing NTPase fold" is made up of an 8-stranded twisted $\beta$-sheet flanked by $\alpha$-helices. The 58 -residue $\mathrm{C}$ terminal domain consisting of three helices and three $\beta$-strands also protrudes out of the rest of the structure and is known to play a key role in forming higher order aggregation of the protein filaments into bundles. The $M$ domain in RecA, comprising the P-loop-containing NTPasefold has several biologically important loop regions. The P-loop region comprising 67-74 is important for nucleotide binding while the loop in the adjacent strands (residues 225-241) is known to be involved in LexA binding and SOS response. ${ }^{19,20}$ Two loops L1 (residues 157-165) and L2 (residues 195-210), are known to be responsible for recognizing DNA in the RecA filament. ${ }^{21,22} \mathrm{~L} 2$ is also believed to be involved in ATP-mediated allosteric changes.

The overall structures in all the complexes studied here are highly similar to the previously reported MtRecA apo structure ${ }^{7}$ with r.m.s. deviations for the whole molecule as well as for the individual domains well within $1 \AA$. The $\mathrm{N}$ domain exhibits the least changes in all the four structures, while the $\mathrm{M}$ domain containing the nucleotide 
TABLE I. Data Collection and Refinement Statistics ${ }^{\dagger}$

\begin{tabular}{|c|c|c|c|c|}
\hline & RecA-Adp & $\begin{array}{l}\text { RecA- } \\
\text { Atp } \gamma \mathrm{S}\end{array}$ & RecA-Atp $\gamma \mathrm{S}-\mathrm{Mg}^{+2}$ & RecA-dATP-Mg ${ }^{+2}$ \\
\hline Space group & $\mathrm{P} 6_{1}$ & $\mathrm{P} 6_{1}$ & $\mathrm{P} 6_{1}$ & $\mathrm{P} 6_{1}$ \\
\hline \multirow[t]{2}{*}{ Unit cell dimensions $(\AA)$} & $\mathrm{a}=108.5$ & $\mathrm{a}=108.1$ & $\mathrm{a}=108.0$ & $\mathrm{a}=108.1$ \\
\hline & $\mathrm{c}=72.9$ & $\mathrm{c}=72.5$ & $\mathrm{c}=72.5$ & $c=72.4$ \\
\hline $\mathrm{Z}$ & 6 & 6 & 6 & 6 \\
\hline Solvent content (\%) & 61.5 & 61.1 & 61.0 & 60.9 \\
\hline Resolution $(\AA)$ & 3.10 & 3.20 & 3.25 & 3.20 \\
\hline Last shell $(\AA)$ & $3.21-3.10$ & $3.31-3.20$ & $3.37-3.25$ & $3.31-3.20$ \\
\hline Total number of measurements & 54,593 & 40,215 & 30,714 & 60,221 \\
\hline Total number of unique reflections & 8,813 & 8,054 & 7,435 & 8,055 \\
\hline Number of reflection with $\mathrm{I} \leq 0$ & 364 & 523 & 382 & 358 \\
\hline Completeness of data $(\%)$ & $97.9(96.8)$ & $99.7(99.5)$ & $96.6(95.9)$ & $99.9(100.0)$ \\
\hline $\mathrm{R}_{\text {merge }}(\%)$ & $8.3(33.4)$ & $10.8(33.0)$ & $9.3(31.4)$ & $10.3(32.7)$ \\
\hline Average $\mathrm{I} / \sigma(\mathrm{I})$ & $7.8(2.0)$ & $6.5(2.1)$ & $7.7(2.9)$ & $6.8(2.1)$ \\
\hline Resolution limit used in refinement $(\AA)$ & $30.0-3.1$ & $30.0-3.2$ & $30.0-3.25$ & $30.0-3.2$ \\
\hline No. reflection with $\mathrm{F}>0$ & 8,449 & 7,523 & 7,053 & 7,691 \\
\hline $\mathrm{R}_{\text {factor }}(\%)$ & 18.9 & 20.4 & 18.7 & 18.9 \\
\hline $\mathrm{R}_{\text {free }}(\%)$ & 24.4 & 25.4 & 27.2 & 24.7 \\
\hline \multicolumn{5}{|l|}{ Average B factor $\left(\AA^{2}\right)$} \\
\hline Main chain & 38.8 & 25.1 & 32.2 & 19.7 \\
\hline Side chain & 42.2 & 29.8 & 37.6 & 27.6 \\
\hline \multicolumn{5}{|l|}{ R. M. S. deviation from ideal } \\
\hline Bond length $(\AA)$ & 0.006 & 0.008 & 0.007 & 0.007 \\
\hline Bond angles $\left(^{\circ}\right)$ & 1.1 & 1.8 & 1.7 & 1.2 \\
\hline Dihedral angles $\left(^{\circ}\right)$ & 22.1 & 23.2 & 22.8 & 22.3 \\
\hline Improper angles $\left(^{\circ}\right)$ & 0.7 & 1.2 & 1.2 & 0.8 \\
\hline Number of protein atoms & 2,318 & 2,354 & 2,313 & 2,285 \\
\hline Number of ligand atoms & 27 & 31 & 31 & 30 \\
\hline Number of solvent atoms & 79 & 71 & 61 & 57 \\
\hline \multicolumn{5}{|l|}{ Ramachandran plot (\% of residues in the) } \\
\hline Allowed regions & 98.1 & 95.6 & 96.3 & 97.7 \\
\hline Generously allowed regions & 1.1 & 3.2 & 3.7 & 1.9 \\
\hline Disallowed regions & 0.8 & 1.2 & 0.0 & 0.4 \\
\hline
\end{tabular}

'Values in parentheses refer to the highest resolution shell.

binding site showed subtle but noticeable structural variations. The sequences of two surface-exposed loops (32-36 and 232-236), which show the largest deviations, are highly variable among bacterial species. Significant variation was also observed at the helix succeeding L2, particularly in residues 211-212. Despite the modest resolution limits to which the crystals diffracted, electron density was well defined for most side chains, particularly those involved in hydrogen-bond and salt-bridge interactions. Upon analysis of all MtRecA structures, three hydrogen bonds between pairs of side chain atoms appeared to be invariant, of which the one between $75 \mathrm{Thr}$ OG1 and $104 \mathrm{Tyr} \mathrm{OH}$ is in the nucleotide binding site. Salt bridges between residue pairs 223-264, 242-277, and 131-135, involved in inter-strand, inter-domain, and intra-helical stabilization, respectively, are found to be invariant in all MtRecA as well as in Escherichia coli RecA (EcRecA) structure. It is interesting to note that residues Arg 223 and Glu 64 are conserved in most of the eubacterial RecAs, while residues Asp 131 and Arg 135 are conserved in many of them. ${ }^{23}$ High conservation index of these residues perhaps reflects the crucial role of these salt bridges in maintaining the required structure of the $\mathrm{M}$ domain, considered as the "business" part of the RecA molecule.

\section{Filaments and Bundles}

Electron microscopy studies of EcRecA have clearly shown that the active conformation of RecA is a nucleoprotein filament. The crystal structures of RecA from E. coli, ${ }^{21}$ and that from M. tuberculosis ${ }^{7}$ have also shown that the protein in the crystals polymerises into similar filaments. The filaments of RecA further aggregate to form bundles, which is again observed both in the crystal structures and in the electron micrographs. We have shown recently that the association of filaments into bundles is much weaker in MtRecA as compared to that in $\operatorname{EcRecA}^{7,24}$ and have also discussed its possible implications for recombination in mycobacteria. Recent studies using electron microscopy and plasmon resonance techniques ${ }^{5}$ have indicated that ATP promotes the high-affinity ssDNA binding state by increasing the cooperative association of adjacent RecA subunits in a filament rather than increasing the inherent affinity of the RecA monomer for ssDNA. Therefore, it is of interest to study the structural details at the interface between two RecA monomers in a filament, both in the presence and absence of nucleotides. The filament and bundle formation in all the complexes is very similar to that in apo MtRecA except for a small but significant 

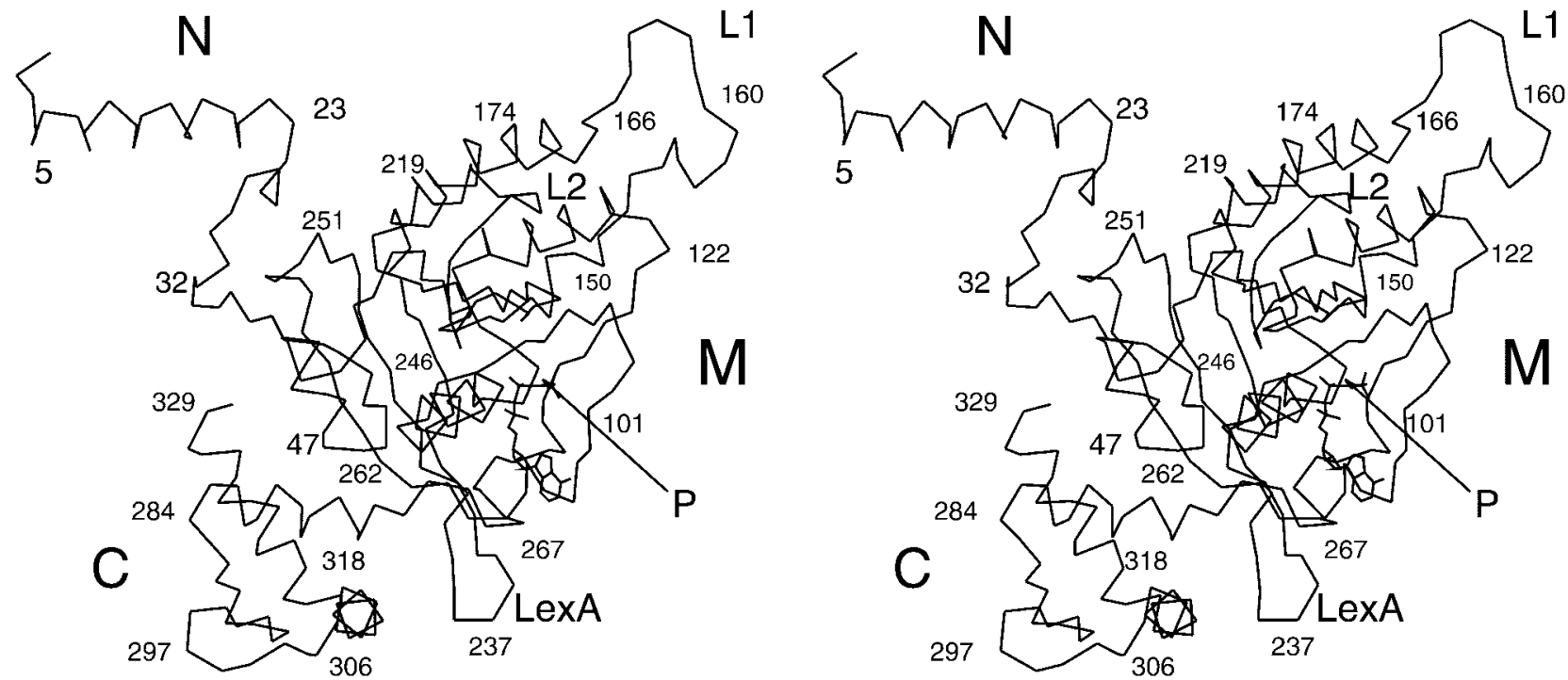

Fig. 1. Stereoview of the $C_{\alpha}$ plot of RecA. Several residues are numbered. The three domains are marked N, M, and C. L1 and L2 refer to the DNA binding loops. Loop L1 is derived from the MtRecA-ATP $\gamma \mathrm{S}-\mathrm{Mg}^{+2}$ complex whereas the loop L2 is derived from the MtRecA-ATP $\gamma \mathrm{S}$ complex. The P-loop and the LexA binding loop are also indicated.

variation in the ADP complex. The residues involved in filament formation are situated at the $\mathrm{N}$ terminal domain as well as in the 114-135 strand of the M domain and most of them are highly conserved among bacterial RecAs. Nine common hydrogen bonds were found between the monomers in the filaments in all MtRecA structures. Similarly, one hydrogen bond involving residues 36 and 298 was found to be invariant at the filament surface forming the bundle. In the MtRecA-ADP filament, residue pairs 155214, 157-177 are positioned appropriately to form two additional salt bridges. Similarly, Glu 297 from one filament and Lys 24 from the second filament were situated $2.6 \AA$ apart, at the bundle interface, indicating both stronger filament and stronger bundle formation in the presence of $\mathrm{ADP}$.

Two types of RecA filaments have been observed by electron microscopy: filaments with a pitch of $62-72 \AA$ that form in the absence of either nucleic acid or nucleotide cofactors and the $95-\AA$ pitch filaments that form in the presence of both nucleic acid and the nucleotide cofactor. ${ }^{25}$ It is generally believed that RecA molecules are normally stored in the form of bundles by aggregation of inactive filaments and, upon induction of the SOS response, dissociate from the bundles and subsequently form active filaments that take part in recombination and repair. The filaments observed in the crystal structures of both EcRecA and MtRecA with helix pitches of 83 and $72 \AA$, respectively, do not correspond to active filaments in their pitch; nevertheless, they retain the overall nature and several properties of filament formation observed by electron microscopy. Analysis of the MtRecA-ADP complex shows additional interactions both within the filaments as well as between them making both filaments and bundles stronger than in any other MtRecA structure studied here. Biochemical characterization of the RecA protein ${ }^{26}$ suggests that $\mathrm{ADP}$ has an antagonistic effect on ATPase and strand exchange activities and such antagonism might be achieved by strengthening the compaction of filaments into bundles. The subtle structural rearrangements observed in the M-domain of each monomer of the MtRecAADP complex leading to stronger filament and bundle formation corroborate the biochemical data and the hypothesis that emerges out of it. We would like to point out here that the observation of increase in filament-and bundleforming interactions in MtRecA complexes should be distinguished from the weaker bundle formation in the MtRecA compared to EcRecA as the former refers to an increase among MtRecA structures and the latter refers to a difference between MtRecA and EcRecA. Although no detailed data are available at present, an EcRecA-ADP complex may be expected to show a similar increase in filament and bundle strength as compared to that of its apo structure.

Eldin and co-workers have carried out site-directed mutagenesis studies ${ }^{27}$ that lend support to the importance of several residues at the interface in filament formation, deduced from crystal structures. In particular, mutations of residues Lys 6 and Asp 139 that form an inter-monomer salt bridge, as well as mutations of Arg 28 and Asn 113, which are involved in a inter-monomer hydrogen bond, have been reported. These studies indicated that Lys 6 and Arg 28 mutations impose several defects on the oligomeric stability of free RecA protein while permitting normal functioning of RecA-DNA complexes. These observations led them to suggest that the inter-monomer contacts in this region of the protein must be different in free RecA (inactive) and in RecA-DNA (active) nucleoprotein filaments.

On the other hand, the crystal structures of MtRecA and EcRecA clearly show formation of a super-secondary structure where an additional $\beta$ layer is positioned next to the existing $\alpha \beta$ layer forming a hybrid $\beta$ sheet. In a previous 
study, ${ }^{7}$ we carried out molecular modeling studies using the inactive filaments of both EcRecA and MtRecA to obtain 95 - $\AA$ filaments that could possibly reflect the active filament structures. Analysis of the MtRecA and EcRecA crystal structures as well as the modeled $95-\AA$ filaments suggests that the stability conferred by the formation of such a super-secondary structure involving primarily main chain-main chain interactions is far greater than that conferred by individual inter-monomer hydrogen bonds contributed by the side chain atoms of the residues. The experimentally observed $72 / 83 \AA$ (inactive) filaments and the extended 95 - $\AA$ (active) filaments both share this feature whereas a number of additional hydrogen bonds observed in inactive filaments are absent in the extended filaments. Mutations that disrupt the formation of supersecondary structure, such as introduction of a bulky group in the strand made of residues 23-25, can therefore be expected to have a far greater destabilizing effect than individual mutations of the side chains involved in the inter-monomer hydrogen bonds.

\section{Loops Involved in DNA Binding}

The two DNA-binding loops L1 and L2 were not deciphered in EcRecA at all, while only part of L1 was observed in the MtRecA apo structure. Upon binding to ATP $\gamma \mathrm{S}$, however, loop L2 becomes clearly interpretable from the electron density map. In the presence of ATP $\gamma \mathrm{S}$ and magnesium, L1 was clearly interpretable. Thus, the two structures together provide a complete definition of the two loops and, therefore, of the whole protein. The location of these loops in the M-domain is shown in Figure 1. Upon filament formation, these two loops form the surface of the inner core that defines the DNA binding groove [Fig. 2(a)]. The precise orientation and conformation of these loops, defined for the first time, provides a framework for understanding DNA binding by RecA.

Conformational changes upon ligand binding in RecA (primarily in loop L2) to attain high affinity for DNA binding have been expected based on site-directed mutagenesis studies as well as similarity to other proteins in the structural family such as Ras-P21. ${ }^{28}$ Possibilities of such changes in loop L1 have remained unclear. These changes are thought to trigger the conversion of inactive filaments into active nucleoprotein filaments. Unfortunately, conformational changes in the loops could not be understood in detail at this stage, as the two loops are fully defined only in one structure each. Theoretically, the loop in the ATP $\gamma \mathrm{S}$ $-\mathrm{Mg}^{+2}$ complex that provided a clear definition of $\mathrm{L} 1$ can be expected to be in the high-affinity state. Loop L1 has been partly defined in other MtRecA structures, which allows a comparison of those parts of the loop. There appear to be no major changes in L1 when compared to apo MtRecA as well as MtRecA-ADP complex, where 5 out of 8 residues of the loop [Fig. 2(b)] were visible in their electron density maps. These data suggest that major conformational changes in loop L1 are unlikely upon nucleotide binding. Loop L2, on the other hand, is seen clearly in the ATP $\gamma \mathrm{S}$ structure. Comparison with the end residues of loop L2 that were interpretable in other MtRecA structures show that subtle variations exist in this region and in 3-4 residues succeeding the loop [Fig. 2(c)], suggesting that conformational changes upon nucleotide binding are more likely in this loop.

Communication between L1 and L2 has been an important open question as the two loops are far apart in space. Communication from the nucleotide binding site to the loops has also been a question of equal interest. Detailed analysis of the various MtRecA structures reveals complex networks of interactions connecting the two loops with residues involved in nucleotide binding, through a cluster of residues in the vicinity, primarily Val 147 and Ala 148. The main chain atoms of residues 147 and 148 appear to participate in hydrogen bonds with the two regions, 193194 and 211, segments immediately preceding and succeeding L2, respectively. Incidentally, these two residues form a part of the Walker B motif and are totally conserved in all bacterial RecAs as well as in many other P-loopcontaining proteins. ${ }^{29,30}$ Gln 195 at the beginning of L2 by directly hydrogen bonding with the $\gamma$-phosphate oxygens can trigger a conformational change in L2, which can then be transmitted to L1 through the 147-148 segment. This segment can be termed the "connector segment" and could transmit the structural changes to $\mathrm{L} 1$, to render the loop more ordered in the structure.

\section{RecA-Nucleotide Interactions}

The location where the nucleotide binds in MtRecA protein has been defined previously in the crystal structure of the MtRecA-ADP-AlF ${ }_{4}$ complex [Fig. 3(a)], which is essentially the same as that identified by Story and Steitz in the EcRecA-ADP complex. ${ }^{31}$ As compared to that in the EcRecA structure, the P-loop region was shown to be expanded in the apo MtRecA structure, although the amino acid residues in the binding site are substantially conserved in all known RecAs. The expansion of the P-loop observed in the crystal structures of apo MtRecA and the MtRecA-ADP-AlF ${ }_{4}$ complex provided an explanation for the observed biochemical differences in binding and hydrolysis. All the complexes studied here show a similar expansion of the P-loop region, leading to the widening of the nucleotide binding pocket, thus strengthening the previously identified structure-function relationship. The fact that this rearrangement is observed in various MtRecA structures including an apo form, a complex with ADP, as well as complexes with ATP analogues, suggest that the expansion is independent of ligand binding and any associated conformational changes and is probably a speciesspecific strategy for modulating nucleotide binding.

The electron density for the nucleotides and the P-loop regions (residues 67-74) was very well defined in all the complexes, despite modest resolutions to which the crystals diffracted [Fig. 3(b)]. Residues 68, 70-75, 101, 104, $195,228,241,266$ were found to be in interacting distance with the nucleotides. The purine base interacts with Asp 101 and Tyr 104, while the sugar hydroxyls are at the right distance to hydrogen bond with Asn 241, Arg 228, Thr 75, and Tyr 104. The phosphate atoms are surrounded by the P-loop residues and Gln 195, making several interactions. 


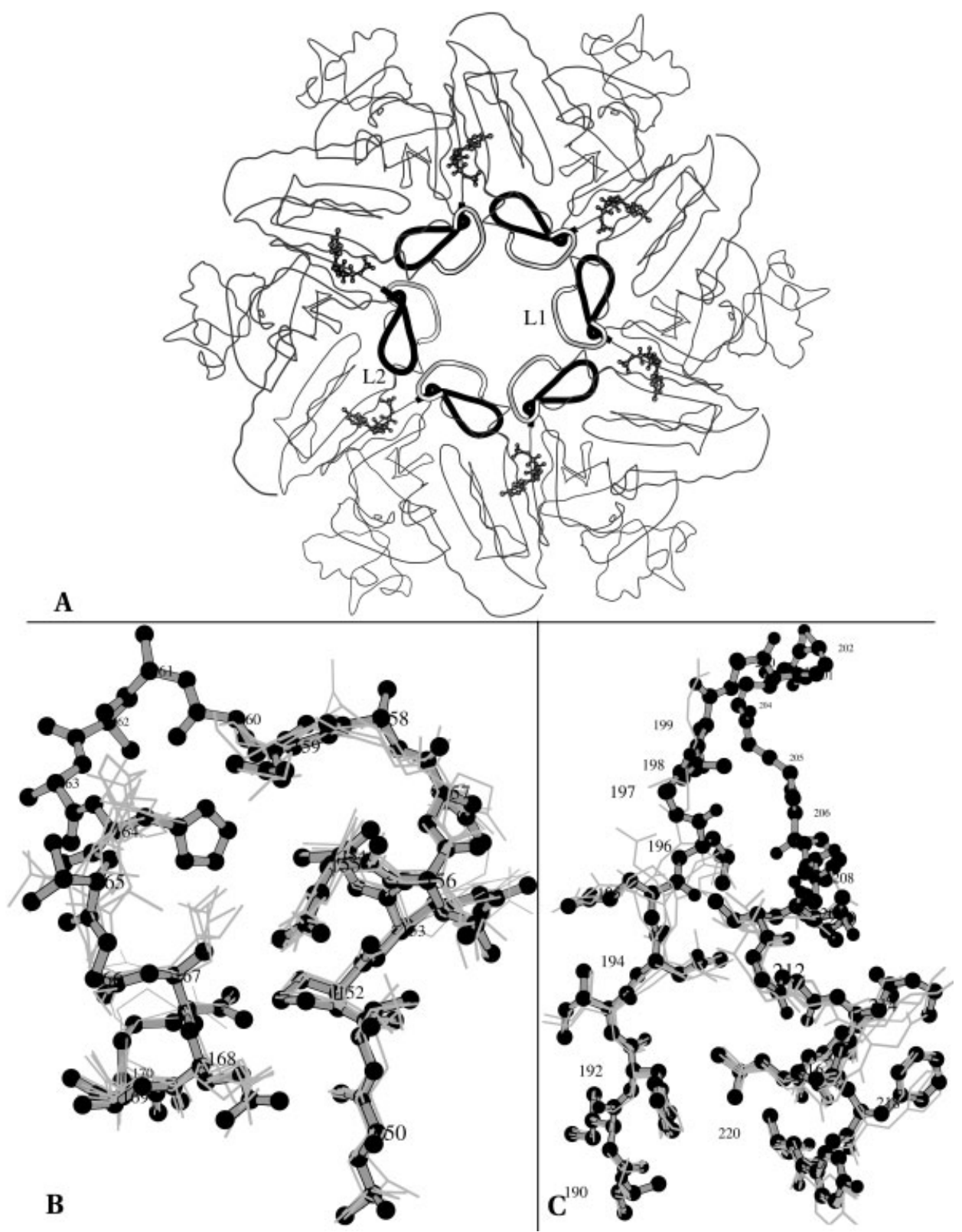

Fig. 2. A: A view down the axis of the MtRecA filament highlighting the residues in the two loops, $L 1$ and $L 2$, that form part of the inner core of the filament. DNA is expected to bind at the groove in the centre. Superposition of the residues corresponding to the loop (and five residues preceeding and five residues succeding the loop) regions (B) L1 and (C) L2, L1 seen clearly in the ATP $y \mathrm{SMg}^{+2}$ complex and L2 seen in ATP $y$ S complex, are shown in black. The loops in the other structures were only partially decipherable from their electron density maps, and are shown in gray shades.

The ATP $\gamma \mathrm{S}$-bound structures reveal the position of the $\gamma$-phosphate as well as the interactions it makes with the protein [Fig. 3(c)]. The terminal phosphate group is surrounded by residues 69,70 , and 195 , probably making as many as five hydrogen bonds. In the case of ATP, an additional hydrogen bond can be expected with residue 69 , owing to the replacement of the - $\mathrm{SH}$ by the $\mathrm{OH}$ group. The conformations of individual nucleotides in all the four complexes were compared in detail. The changes are the largest in the sugar atoms and the least in the base atoms, indicating the stringent requirement in the orientation of the base so as to enable stacking interaction with the side chain of Tyr 104. In all the structures, the anti conformation of the adenine base with respect to the sugar is maintained. Among the four different types of nucleotides, the conformation of $\mathrm{ADP}-\mathrm{AlF}_{4}$ is distinctly different from those of the other three in the phosphate backbone as well as in the sugar moiety. There is no appreciable difference between the two ATP $\gamma \mathrm{S}$ complexes in the conformation or the interactions with the protein. The density for magnesium was not obvious from the electron density map. However, comparison with several other structures indi- 


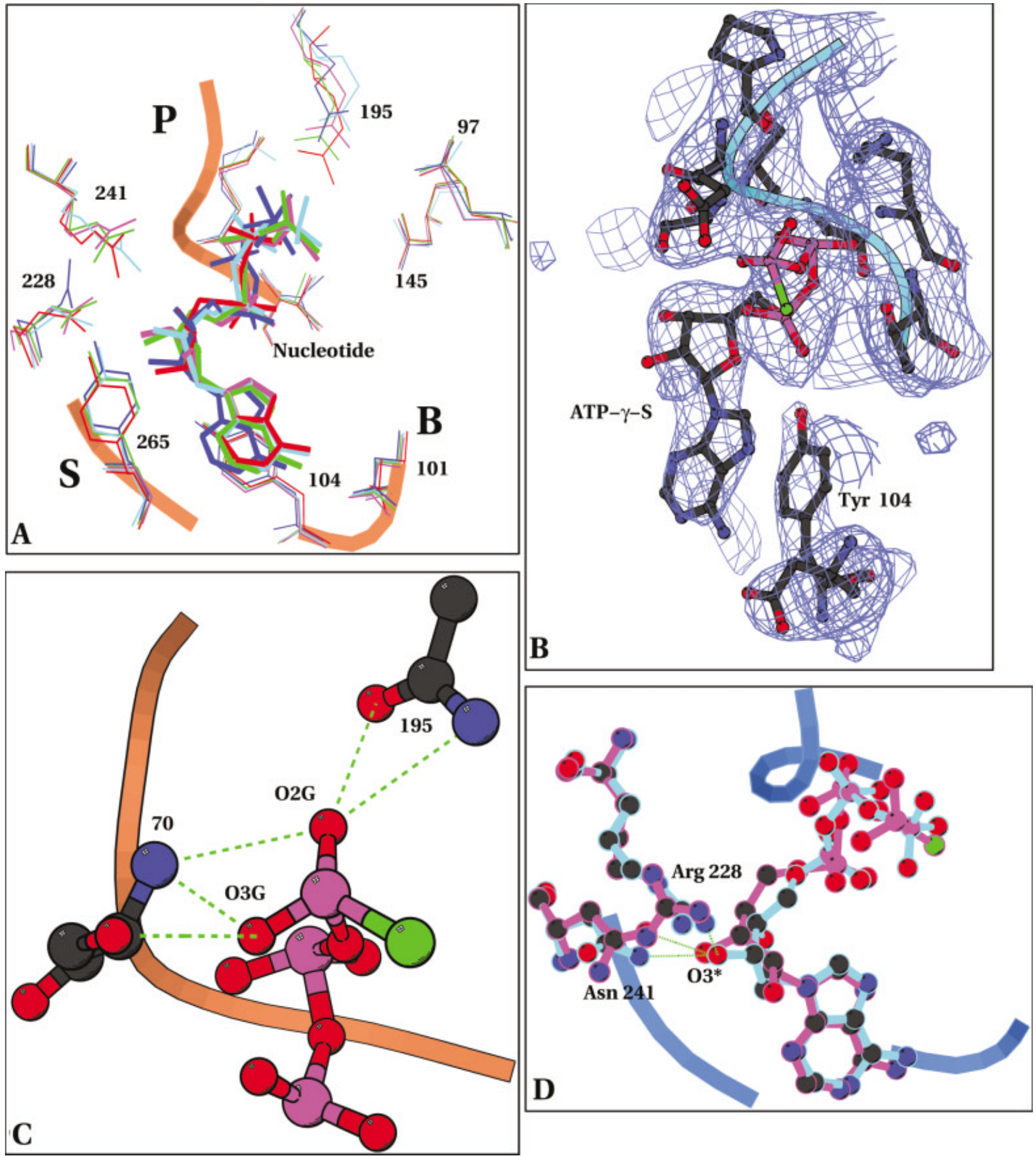

Fig. 3. A: Superposition of the nucleotide binding sites of different MtRecA complexes. The $P, B$, and $S$ regions refer to the residues that bind to phosphate, base, and sugar, respectively. Residues and the nucleotide in the ADP bound structure are shown in red while those in the dATP complex are shown in cyan and those in the ATP $\gamma$ S complexes in the presence and absence of magnesium are shown in magenta and green, respectively. Those in the ADP-AIF ${ }_{4}$ complex are shown in blue. This figure has been prepared using MOLSCRIPT. ${ }^{38}$ B: An Fo-Fc omit map (contoured at $2.5 \sigma$ ) of the nucleotide binding site in the MtRecA-ATP $\mathrm{S}$ complex. Stacking interaction of the Tyr-104 is clearly seen. The position of the P-loop is represented by a cyan ribbon. The figure has been prepared using BOBSCRIPTT. ${ }^{39} \mathbf{C}$ : Interaction of the terminal phosphate of ATP $\gamma \mathrm{S}$ with the protein. Hydrogen bonds are indicated by green dotted lines. D: Superposition of ATP $\gamma S$ (magenta) and dATP (cyan) complexes of MtRecA. Nucleotides and residues Arg 228 and Asn 241 are shown in ball-and-stick representation. The O3' sugar hydroxyl in dATP structure superposes on the O2' sugar hydroxyl of ATP $\gamma$ S.

cates that magnesium could occupy a position close to the $\beta$ - and the $\gamma$-phosphates, held in place also by the side chain of Thr-74 and a water molecule hydrogen-bonded to
Asp-145. Comparison of the ATP $\gamma \mathrm{S}$ and $\mathrm{ADP}-\mathrm{AlF}_{4}$ complexes, both analogues of ATP, reveals that the position of the terminal $\gamma \mathrm{S}$-phosphate moiety does not coincide pre- 
cisely with that of the $\mathrm{AlF}_{4}$ moiety. In fact, $\mathrm{AlF}_{4}$ appears to be closer to the $\beta$-phosphate of ADP and ATP $\gamma \mathrm{S}$ as well as to the free phosphate seen in the apo MtRecA structure. This, together with the fact that the number of interactions made by $\mathrm{AlF}_{4}$ with the $\alpha$ and $\beta$ phosphates in the $\mathrm{ADP}-\mathrm{AlF}_{4}$ complex are fewer than in the case of the MtRecA-ATP $\gamma \mathrm{S}$ complexes, suggests that $\mathrm{ADP}-\mathrm{AlF}_{4}$ is structurally a sub-optimal analogue of ATP.

Differences are observed between the ATP $\gamma \mathrm{S}$ and dATP structures as well, especially in the conformation and the relative orientation of the sugar moieties with respect to the base and the phosphates. It is interesting to note that a close approach by the $2^{\prime}$ hydroxyl of ATP $\gamma \mathrm{S}$ to the side chain of Asn-241 (4.2 $\AA$ ) is replaced by a possible hydrogen bond (3.3 $\AA$ ) by the $3^{\prime}$ hydroxyl in dATP [Fig. 3(d)]. The difference in the sugar conformation reflects the adaptation required by the nucleotide to maintain a similar network of interactions responsible for nucleotide binding. Residue 241 in EcRecA is a serine instead of asparagine in MtRecA. The serine side chain being smaller is too far removed from the sugar hydroxyl to make a strong hydrogen bond. Therefore, it can be expected that the affinity of EcRecA to dATP cannot be regulated by this residue. Biochemical data ${ }^{32}$ indicate that EcRecA can bind dATP albeit with a slightly reduced affinity. In EcRecA, Try 264 is said to make a hydrogen bond with the O3' hydroxyl of ADP and presumably with that of ATP as well. It can, therefore, be expected that dATP requires to change in conformation when bound to EcRecA. Tyr 264 is shown by mutagenesis and cross-linking ${ }^{33}$ studies also to be important for nucleotide binding. In EcRecA, this residue was found to interact with the nucleotide. ${ }^{31}$ In MtRecA, however, the corresponding tyrosine (Tyr 265) does not interact with the nucleotide in any of the complexes, indicating that subtle differences in binding site geometry that exist between proteins from these two species could play a major role in bringing about finer differences in binding affinities and specificities.

The amino acid residues in the binding pocket in the different complexes superpose well, despite differences in the conformation of the different nucleotides, reflecting the relative rigidity of the pocket [Fig. 3(a)]. The highest deviations, which are less than $0.7 \AA$, are observed in residues 69,70 , and 195 . As indicated in an earlier sequence analysis study, ${ }^{34} 69$ and 70 are not stringent residues for RecA to be active and hence their structural alteration is not quite unexpected. Only one side chain-side chain interaction, Thr 75 OG1-Tyr 104 $\mathrm{OH}$, was found to be invariant over all the structures including apo EcRecA and MtRecA. Three additional interactions, two main chain-side chain (68 O-Lys 73 NZ; 69 N-Gln 195NE2) and one side chain-side chain (Glu 69 OE1-Ser 70 OG) interactions, were found in EcRecA. Small differences in interaction patterns observed between EcRecA and MtRecA are reflected in the differences in the geometries at the nucleotide binding clefts in the two structures.

\section{Correlation With Site-Directed Mutagenesis Studies Involving the P-Loop}

The importance of structural rigidity of the binding site is emphasized by the results of the site-directed mutagenesis studies of the P-loop residues, as well. Extensive biochemical data have been reported, ${ }^{35}$ where every residue of the P-loop motif of EcRecA has been systematically mutated and the effect of mutation on function studied. A high degree of conservation of the sequence and structure of the P-loop and neighbouring motifs between EcRecA and MtRecA make the mutagenesis data for EcRecA relevant to MtRecA as well. To understand the precise changes that might occur in the active sites of these mutants, 21 mutants sampled across the range of reported biochemical activities such as those with full partial, and no detectable activities were chosen for analysis (Table II). Structures of these mutants in both EcRecA and MtRecA were built and energy minimised based on the respective crystal structures, using standard homology modeling procedures. Analysis of these structures provide reasonable explanations for the observed biochemical behavior as described below.

\section{Gly-67, Gly-72, Lys-73, and Thr-74}

These residues define the consensus sequence of the P-loop motif. Biochemical studies indicate that even conservative mutations at these positions result in total loss of activity. ${ }^{35}$ The $\phi$ and $\Psi$ torsion angles $\left(158^{\circ}, 176^{\circ}\right)$ observed at position 67 can only be satisfied by glycine, explaining its strict requirement. Mutating this to an alanine would result in a significant shift in the 67-74 loop, making it unable to retain the interactions with the nucleotide. Gly-72, when replaced by any other residue, introduces short contacts with other residues. Lys-73 not only interacts with the $\alpha$ and $\beta$ phosphate oxygens, but also with the side chain of Gln-195. An arginine in this position will have its side chain pointing away from the phosphates, perhaps stabilized by new salt bridges with Glu-97 and Asp-145, both conserved residues, the latter in fact part of the Walker-B motif, thus leading to a simultaneous loss their availability for nucleotide binding. A threonine at this position not only causes loss of interactions with the nucleotide but also leads to a shift in Gln-195 and Thr-74, possibly disturbing the position of magnesium in the process. The side chain of Thr-74 makes a strong hydrogen bond with Asp-145. Replacing it with an alanine or isoleucine would abolish this hydrogen bond, leading to the disturbance of the binding site and loss of activity. The subtle structural changes observed in the mutants of any of the four residues appear to be sufficient to result in total loss of nucleotide binding, correlating with the biochemical observations.

\section{Pro 68, Glu-69, Ser-70, Ser-71, and Thr-75}

These residues form the nonconsensus part of the motif since they are not strictly required to define it. However, they have been found to be essential for full activity, with the exception of Pro-68, which can tolerate changes into a range of polar and nonpolar residues. It is interesting, 
TABLE II. Structure-Function Correlation of the 21 P-Loop Mutants Used in This Study

\begin{tabular}{|c|c|c|c|}
\hline \multirow[b]{2}{*}{ Mutant } & \multirow[b]{2}{*}{ Phenotype } & \multicolumn{2}{|c|}{ Interaction with } \\
\hline & & Ligand & Protein residue \\
\hline G67A & $\mathrm{rec}^{-}$ & None & 67,73 \\
\hline P68R & $\mathrm{rec}^{+/-}$ & None & $69,71,198$ \\
\hline E69D & $\mathrm{rec}^{+/-}$ & None & 71,197 \\
\hline E69A & $\operatorname{rec}^{+/-}$ & None & 71 \\
\hline $\mathrm{S} 70 \mathrm{C}$ & $\operatorname{rec}^{+1-}$ & Phosphate & 241 \\
\hline $\mathrm{S} 71 \mathrm{Y}$ & $\mathrm{rec}^{-}$ & None & 68,243 \\
\hline G72D & $\mathrm{rec}^{-}$ & Phosphate & 73,76 \\
\hline G72V & $\mathrm{rec}^{-}$ & None & \\
\hline K73G & $\mathrm{rec}^{-}$ & Phosphate & 77 \\
\hline $\mathrm{K} 73 \mathrm{R}$ & $\mathrm{rec}^{-}$ & Phosphate & $65,77,193$ \\
\hline K73N & $\mathrm{rec}^{-}$ & Phosphate & 77,73 \\
\hline $\mathrm{T} 74 \mathrm{~S}$ & $\mathrm{rec}^{-}$ & Phosphate & 78 \\
\hline $\mathrm{T} 74 \mathrm{P}$ & $\mathrm{rec}^{-}$ & None & 78 \\
\hline $\mathrm{T} 74 \mathrm{I}$ & $\mathrm{rec}^{-}$ & None & 72,78 \\
\hline $\mathrm{T} 75 \mathrm{M}$ & $\mathrm{rec}^{+/-}$ & Sugar & 72,79 \\
\hline $\mathrm{T} 75 \mathrm{~L}$ & $\operatorname{rec}^{+1-}$ & Phosphate & 79 \\
\hline T75A & $\operatorname{rec}^{+/-}$ & Phosphate & 79 \\
\hline T75G & $\mathrm{rec}^{+/-}$ & Phosphate & 79 \\
\hline $\mathrm{T} 75 \mathrm{~V}$ & $\mathrm{rec}^{+/-}$ & Phosphate & 78 \\
\hline T75R & $\mathrm{rec}^{-}$ & None & 72,78 \\
\hline T76R & $\mathrm{rec}^{-}$ & None & 73 \\
\hline
\end{tabular}

however, that introduction of charged residues such as an arginine at this position results in unique properties including discrimination between recombination and repair activities. ${ }^{19}$ The model of the P68R mutant indicates that the side chain, while pointing away from the nucleotide and hence not altering ATPase activity, will form part of the DNA binding groove upon filament formation, thus acquiring a potential to influence the mode of DNA binding. Residues 69 and 70 interact with each other through their side chains in EcRecA, in fact, holding Ser 70 in a sterically unfavorable region, which may be essential to maintain residues 70-72 in an optimal position for nucleotide binding. Modeling studies indicate that a E69G or E69A mutation results in a shift of residues 66-69 as well as the magnesium ion, while E69N and E69D mutations abolish the hydrogen bond with Ser-70, all eventually leading to loss of some interactions with the nucleotide, thus correlating with observed partial loss of activity. At position 70, a glycine can be tolerated with full activity, not surprisingly because glycine is the only residue that can adopt the unusual $\phi / \psi$ angles required at this position without any external influence, while the phosphate interactions can be possibly compensated by the other groups of the pocket. In the MtRecA structure, the 69-70 hydrogen bond does not exist and the whole of the P-loop pocket is expanded because of the shift in the location of the P-loop, which has been correlated with reduced activity. It is also interesting to note that the dihedral angles $\phi$ and $\psi$ of Ser $70(38,80)$ are in a fully allowed region in MtRecA, owing to the changes in the position of the loop resulting in the binding site expansion. Mutagenesis data are not available for MtRecA, but it can be expected that this position can tolerate substitution with any polar residue without loss of activity, unlike in EcRecA. Ser-71 makes several

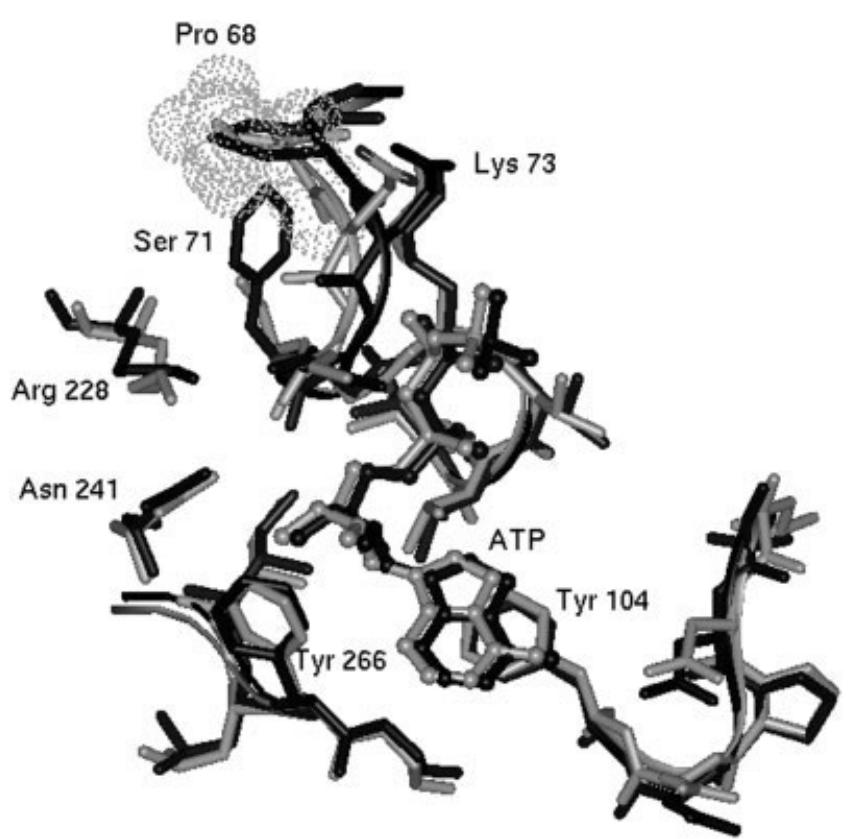

Fig. 4. An example of structural changes in the nucleotide binding pocket in MtRecA upon mutagenesis (see text). Steric clashes within the protein that arise due to the 71 Ser to Tyr mutation are shown as a surface representation. The protein atoms are shown in gray while atoms in the models of mutants are shown in black.

interactions with the phosphates of ATP. Replacing this by a tyrosine induces severe short contacts within the binding pocket (Fig. 4). The total loss of activity in the S71Y mutant can thus be easily explained. Although Thr-75 is just outside the P-loop, it interacts with the $\gamma$ phosphate of ATP, main chain atoms of residue 78 and 79, side chain of Tyr 104 as well as the sugar in ATP. It is conserved in most RecAs as well as in many P-loop containing proteins. An alanine at this position would not be able to retain the interactions with the nucleotide, thus explaining the partial loss of activity. A T75R mutation, on the other hand, would result in a significant change in the P-loop to accommodate the larger side chain, explaining the total loss of activity.

It is clear from the above discussion that loss of activity in the various mutants is generally caused by structural alterations in the nucleotide binding site. This again points to the inherent rigidity of the site or, in other words, the need for the precise arrangement of residues at the binding site for maintaining optimal activity.

\section{Structural Signature of NTP Recognition}

The $\mathrm{M}$ domain of MtRecA, containing the nucleotide binding site, has structural homology with several other proteins that have entirely different functions varying from helicase to involvement in cell cycle regulation, but all with a common nucleotide binding property. A study of these different proteins has recently provided a threedimensional surface motif associated with the P-loop structure. ${ }^{36}$ Here we compare the nucleotide binding in MtRecA with that in a chosen set of representative domains, in 
TABLE III. Structural Similarity and Nucleotide Binding to Structural Neighbours of MtRecA ${ }^{\dagger}$

\begin{tabular}{|c|c|c|c|c|c|c|}
\hline PDB & Function & Ligand & Z-score & RMSD & $\begin{array}{c}\text { Number } \\
\text { of aligned } \\
\text { residues }\end{array}$ & $\begin{array}{l}\text { Residues in contact with } \\
\text { base/sugar/phosphate }\end{array}$ \\
\hline 1NG1 & Signal transduction & GDP & 5.5 & 3.9 & 137 & 248/246/Ploop \\
\hline $1 \mathrm{Do} 0$ & Chaperone & ATP & 5.6 & 4.4 & 134 & 18/396/Ploop,256,393 \\
\hline 1JJ7 & Peptide transporter & $\mathrm{ADP}$ & 5.9 & 3.4 & 126 & 512/515,546/Ploop \\
\hline $1 F N N$ & Cell division control & $\mathrm{ADP}$ & 6.2 & 10.8 & 143 & 192/204/Ploop \\
\hline 1B0U & Histidine permease & ATP & 7.1 & 3.2 & 131 & 16/19/Ploop \\
\hline $1 \mathrm{E} 32$ & Membrane fusion ATPase & $\mathrm{ADP}$ & 7.7 & 3.5 & 142 & 207,249/384,409/Ploop \\
\hline $1 \mathrm{IQP}$ & Replication factor & $\mathrm{ADP}$ & 8.2 & 4.1 & 132 & 28/20/Ploop \\
\hline $1 \mathrm{G} 6 \mathrm{O}$ & Traffic ATPase & $\mathrm{ADP}$ & 9.2 & 3.3 & 140 & 140/61/Ploop,133 \\
\hline 1CR2 & Gene4 protein & DTP & 18.7 & 2.5 & 174 & 504,535/363/Ploop,465* \\
\hline $6 Q 21$ & Ras protein & GCP & 2.7 & 3.5 & 78 & 119,146/117/Ploop,35*,60* \\
\hline 1GIA & Signal transduction & GSP & 1.9 & 4.3 & 92 & $272,269 / 176,150,151 /$ Ploop, $181^{*}, 46^{*}, 203^{*}$ \\
\hline 3PJR & Helicase & ATP & 6.1 & 4.2 & 134 & $286,16 / 571 /$ Ploop, $287^{*}, 254^{*}$ \\
\hline $1 \mathrm{EFT}$ & Elongation factor & GNP & 3.1 & 5.4 & 96 & $136,139,175,176 / 137,26 /$ Ploop, $62^{*}, 84^{*}$ \\
\hline $1 \mathrm{G} 19$ & RecA & SAP & & & & 104,101,266/241/Ploop,195* \\
\hline
\end{tabular}

${ }^{\dagger}$ Residues with * are involved in interaction with $\gamma$-phosphate. ADP, GDP: Adenosine and guanosine di-phosphate, respectively; ATP, GTP: Adenosine and guanosine tri-phosphate, respectively; DTP: deoxy adenosine triphosphate; SAP, GSP: ATP $\gamma$ S and GTP $\gamma$ S, respectively; GCP: guanosyl-methylene tri-phosphate; GNP: guanosyl-imido tri-phosphate.

terms of the location of the binding site as well as the modes of nucleotide binding. A DALI search for structural similarity of the M domain of MtRecA indicates 31 proteins to be structurally similar with $\mathrm{Z}$ scores $^{37}$ greater than 5.0. We chose those hits from the search list that (1) contained a nucleotide bound to it and (2) showed an alignment length that was at least $50 \%$ of the length in the $\mathrm{M}$ domain. A nonredundant list using the above criteria yielded a set of 10 proteins, which are listed in Table III along with their functions, brief structural classifications, and the types of nucleotide to which they bind. Apart from these 10, we also chose ras-p21, Ef-Tu, and the $\alpha$-subunit of a $\mathrm{G}$ protein, which have been shown earlier to adopt mechanisms of binding and associated conformational changes similar to those in RecA,${ }^{21}$ thus resulting in a total of 14 structures for comparison. A superposition of the corresponding regions in the 14 structures (Fig. 5) indicates that the nucleotide binds in a similar location in all of them. This is despite their sequences bearing no significant similarity except in the two Walker motifs, and their structures having diverse domain organizations. The sequence motifs that are common to all of them, however, correspond to the P-loop or the Walker A motif with a consensus sequence [G/A]XXXXGK[S/T] $]^{30}$ and the Walker $\mathrm{B}$ motif with a consensus sequence $\mathrm{zzz}[\mathrm{I} / \mathrm{L} / \mathrm{F}] \mathrm{Db}$, separated anywhere between 20 and 200 residues from the Walker $\mathrm{A}$ motif. In the Walker B motif, $\mathrm{z}$ stands for hydrophobic residues and $b$ stands for polar residues. They are denoted in lowercase since they are found at these positions in almost all, but not quite all, of the structures studied here and their homologues. Asp 145 in this motif is extensively conserved (Fig. 6) with one exception of replacement with glutamic acid (IG6O). These two motifs together can be said to form a fingerprint for nucleotide tri-phosphate (NTP) recognition. Apart from these two motifs, in RecA, a glutamic acid Glu 97 separated from Walker A motif by 24 residues and a glutamine Gln 195 separated by 50 residues from Walker B motif are observed to be important for

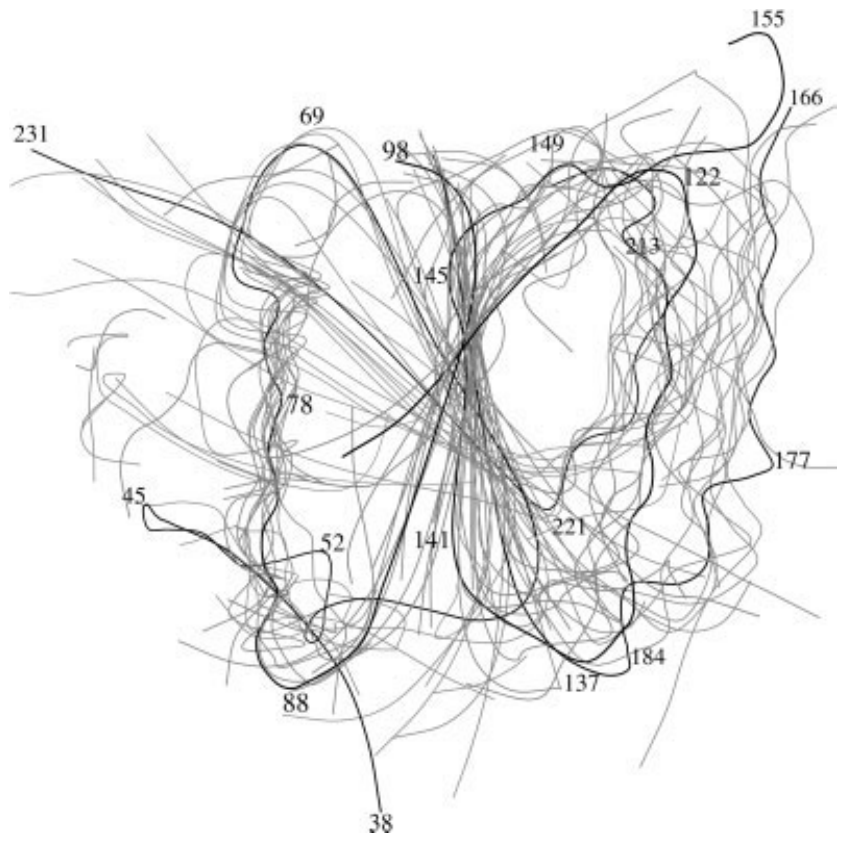

Fig. 5. Superposition of the core of the $\mathrm{M}$ domain (residues 38 to 239) (dark line) and the corresponding regions in the 13 structural neighbours (thin lines). Several residues are numbered.

NTP binding. A corresponding glutamic acid and a glutamine/histidine/threonine are present in most of the structures studied here.

Upon superposition of the whole domains in the various structures, the conserved lysine residue was found to be in excellent alignment, emphasizing its crucial role in recognizing the phosphate oxygens of the nucleotide. The nucleotides themselves also superposed reasonably well in their phosphate atoms. However significant variation was observed in the base and the sugar regions (Fig. 6). A tyrosine residue (Tyr 104) found in both EcRecA and 


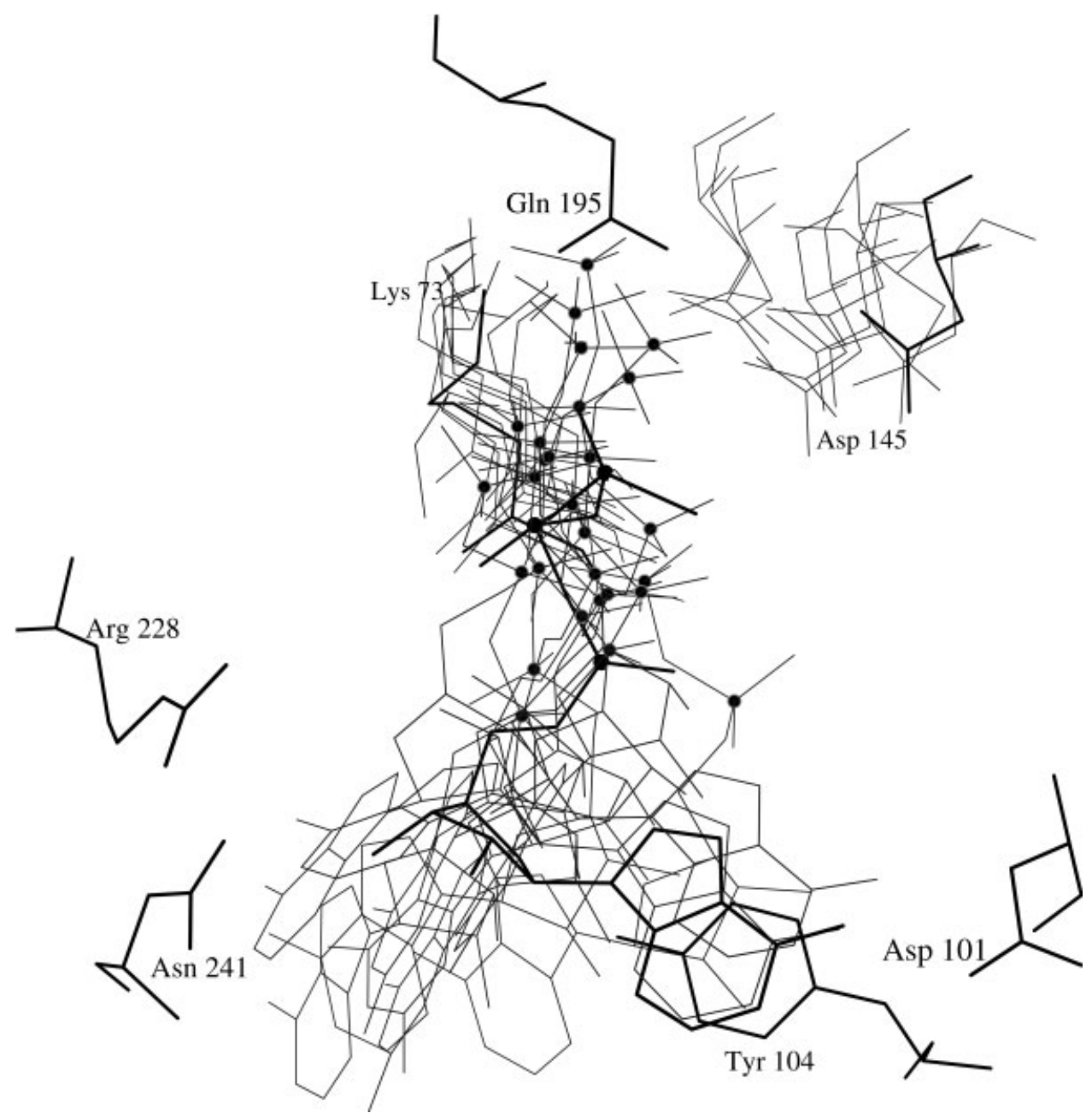

Fig. 6. Positions of the nucleotides in the complexes of MtRecA (black) and its structural neighbours after superposition of regions corresponding to the M-domain. Some key residues in the binding pocket are shown. Residues corresponding to Lys 73 and Asp 145 of MtRecA, which are totally conserved, from the structural neighbours are also shown.

MtRecA, also conserved in all other known RecA sequences, makes a stacking interaction with the adenine base. This interaction is not conserved in all the 14 structures and is, therefore, not crucial for generating nucleotide recognition capability. A number of structures show the main chain atoms of a nearby segment interacting with the base instead of the stacking interaction with the tyrosine side chain. In GTP/GDP binding proteins, the $\mathrm{N} 1$ atom of the guanine base was always found to interact with an aspartic acid side chain and $\mathrm{O} 6$ with main chain atoms. A positively charged residue was also found near the purine base in most of these proteins and a polar residue like an asparagine in the rest of them. This residue was found to make a hydrogen bond with a sugar hydroxyl group, in all the structures. GTP/GDP binding proteins appear to have a higher preference for a lysine at this position. The phosphate groups are found to interact with residues in the Walker A or the P-loop motif, with the $\gamma$-phosphate making additional contacts with other residues. In MtRecA, Gln 195 is seen to make hydrogen bonds with the $\gamma$-phosphate oxygens, but in other structures, an Arg/Lys/His or Thr are observed in this position.

The above analysis reveals that the nucleotide binding site is situated in a similar place in all the structures examined here, as also their modes of binding maintained, despite significant differences in sequences and lengths of the domain. A strong relation between fold and function can thus be established, fold referring to P-loop containing NTPase fold and function referring to nucleotide binding.

\section{CONCLUSION}

The complexes reported here clearly identify the interactions of the nucleotide, especially that of the terminal phosphate moiety, with the protein. The two ATP $\gamma \mathrm{S}$ complexes together provide a definition of the two DNA binding loops and provide a framework for exploring the conformational changes that would take place upon nucleotide binding. The structures also show how dATP can be accommodated in the nucleotide binding pocket and maintain affinities to the protein similar to that exhibited by 
ATP. Analysis of the models of the various site-directed mutants of the P-loop region explains the structural role of each residue in the pocket and indicates why the conserved residues cannot tolerate any substitutions, without losing activity. Comparison with structural neighbours confirms that the sequence motifs [G/A]XXXXGK[S/T] and zzz[I/L/ F]Db define a signature for NTP recognition. The conformation and the position of the three phosphate moieties in various structures were found to be superimposable despite significant variation in the orientation of the base and the sugar groups. The nucleotide specificity of the protein is largely reflected in the conformation and mode of binding of the base region.

\section{ACKNOWLEDGMENTS}

Diffraction data were collected on an imaging plate detector at the X-ray Facility for Structural Biology supported by the Department of Science and Technology (DST) and the Department of Biotechnology (DBT). Use of facilities at the Super Computer Education and Research Centre, the Distributed Information Centre, and the Interactive Graphics facility, the latter two supported by DBT is acknowledged. S.D. is a CSIR senior research fellow.

\section{REFERENCES}

1. Kowalczykowski SC, Dixon DA, Eggelston AK, Lauder SD, Rehrauer WM. Biochemistry of homologous recombination in Escherichia coli. Microbiol Rev 1994;58:401-465.

2. Yu X, Egelman EH. Structural data suggest that the active and inactive filaments are not simply interconvertabe. J Mol Biol 1992;227:334-346.

3. Menetski JP, Bear DG, Kowalczykowski SC. Stable DNA heteroduplex formation catalyzed by the Escherichia coli RecA protein in the absence of ATP hydrolysis. Proc Natl Acad Sci USA 1990;87:21-25.

4. Konforti BB, Davis RW. ATP hydrolysis and the displaced strand are two factors that determine the polarity of RecA-promoted DNA strand exchange. J Mol Biol 1992;227: 38-53.

5. De jutter JK, Forget AL, Logan KM, Knight KL. Phe217 regulates the transfer of allosteric information across the subunit interface of the RecA protein filament. Structure 2001;9:47-55.

6. Logan KM, Forget AL, Verderese JP, Knight KL. ATP-mediated change in cross-subunit interactions in the RecA protein. Biochemistry 2001;40:11382-11389.

7. Datta S, Prabu MM, Vaze MB, Ganesh N, Chandra NR, Muniyappa K, Vijayan M. Crystal structures of Mycobacterium tuberculosis RecA and its complex with $\mathrm{ADP}-\mathrm{AIF}_{4}$ : implications for decreased ATPase activity and molecular aggregation. Nucleic Acids Res 2000;28:4964-4973.

8. Kumar RA, Vaze MB, Chandra NR, Vijayan M, Muniyappa K. Functional characterization of the precursor and spliced forms of the RecA protein of M. tuberculosis. Biochemistry 1996;35:1793-1802.

9. Otwinowski Z, Minor W. Processing of X-ray diffraction data collected in oscillation mode. Methods Enzymol 1997;276:307-326.

10. Berman HM, Battistuz T, Bhat TN, Bluhm WF, Bourne PE, Burkhardt K, Feng Z, Gilliland GL, Iype L, Jain S, Fagan P, Marvin J, Padilla D, Ravichandran V, Schneider B, Thanki N, Weissig H, Westbrook JD, Zardecki C. The protein data bank. Acta Crystallogr D 2002;58:899-907.

11. Brunger AT, Adams PD, Clore GM, DeLano WL, Gros P, GrosseKunstleve RW, Jiang JS, Kuszewski J, Nilges M, Pannu NS, Read RJ, Rice LM, Simonson T, Warren GL. Crystallographic and NMR system (CNS): a new software for macromolecular structure determination. Acta Crystallogr D 1998;54:905-921.

12. Vijayan M. In: Diamond R, Ramaseshan S, Venkateshan K, editors. Computing in crystallography. Bangalore: Indian Academy of Sciences; 1980. p 19.01-19.26.

13. Jones TA. A graphic model building and refinement system for macromolecules. J Appl Crystallogr 1978;11:268-272.

14. Kleywegt GJ, Jones TA. Databases in protein crystallography. Acta Crystallogr D 1998; 54:1119-1131.
15. Laskowski RA, MacArthur MW, Moss DS, Thronton JM. PROCHECK: a program to check the streochemical quality of protein structures. J Appl Crystallogr 1993;26:283-291.

16. Connolly ML. The molecular surface package. J Mol Graph 1993;11:139-141.

17. Cohen GE. ALIGN: a program to superimpose protein coordinates, accounting for insertions and deletions. J Appl Cryst 1997;30:11601161.

18. Murzin AG, Brenner SE, Hubbard T, Chothia C. SCOP: a structural classification of protein database for the investigation of sequences and structures. J Mol Biol 1995; 247:536-540.

19. Dutreix M, Moreau PL, Bailone A, Galibert F, Battista JR, Walker GC, Devoret R. New recA mutations that dissociate the various RecA protein activities in Escherichia coli provide evidence for an additional role for RecA protein in UV mutagenesis. J Bacteriol 1989;171:2415-2423.

20. Ogawa H, Ogawa T. Regulation in repressor inactivation by RecA protein. Adv Biophys 1990;26:33-49.

21. Story RM, Weber IT, Steitz TA. The structure of the E. coli RecA protein monomer and polymer: Nature 1992;355:318-325.

22. Kawashima H, Horii T, Ogawa T, Ogawa H. Functional domains of Escherichia coli RecA protein deduced from the mutational sites in the gene. Mol Gen Genet 1984;193: 288-292.

23. Karlin S, Brocchiei L. Evolutionary conservation of recA genes in relation to protein structure and function. J Bacteriol 1996;178: 1881-1894.

24. Vaze MB, Muniyappa K. RecA protein of Mycobacterium tuberculosis possesses $\mathrm{pH}$-dependent homologous DNA pairing and strand exchange activities: implications for allele exchange in mycobacteria. Biochemistry 1999;38:3175-3186.

25. Egelman EH. What do X-ray crystallographic and electron microscopic structural studies of the RecA protein tells us about recombinations. Curr Opin Struct Biol 1993; 3:189-197.

26. Lee JW, Cox MM. Inhibition of recA protein promoted ATP hydrolysis. ATP gamma S and ADP are antagonistic inhibitors. Biochemistry 1990;29:7666-7676.

27. Eldin S, Forget AL, Lindenmuth DM, Logan KM, Knight KL. Mutations in the N-terminal region of RecA that disrupt the stability of free protein oligomers but not RecA-DNA complexes. $J$ Mol Biol 2000;299:91-101.

28. Milburn MV, Tong L, Devos AM, Brunger A, Yamaizumi Z, Nishimura S, Kim S. Molecular switch for signal transduction: Structural difference between active and inactive forms of protooncogenic ras proteins. Science 1990;347:939-945.

29. Saraste M, Sibbald PR, Wittinghofer A. The P-loop-a common motif in ATP- and GTP-binding proteins. Trends Biochem Sci 1990;15:430-434.

30. Walker JE, Saraste M, Runswick MJ, Gay NJ. Distantly related sequences in the alpha-and beta-subunits of ATP synthase, myosin kinase and other ATP-requiring enzymes and common nucleotide-binding fold. EMBO J 1982;1:945-951.

31. Story RM, Steitz TA. Structure of the RecA protein: ADP complex. Nature 1992;355: 374-376.

32. Watanabe R, Masui R, Mikawa T, Takamatsu S, Kato R, Kuramitsu S. Interaction of Escherichia coli RecA protein with ATP and its analogues. J Biochem 1994;116:960-966.

33. Knight KL, McEntee K. Affinity labeling of a tyrosine residue in the ATP binding site of the recA protein from Escherichia coli with 5 '-p-fluorosulfonylbenzoyladenosine. J Biol Chem 1985;260:1017710184.

34. Roca AI, Cox MM. The RecA protein: structure and function. Crit Rev Biochem Mol Biol 1990;25:415-456.

35. Logan KM, Knight KL. Mutagenesis of the P-loop motif in the ATP binding site of the RecA protein from Escherichia coli. J Mol Biol 1993;232:1048-1059.

36. Via A, Ferre F, Brannetti B, Valencia A, Helmer-Citterich M. Three-dimensional view of the surface motif associated with the P-loop structure: cis and trans cases of convergent evolution. J Mol Biol 2000;303:455-465.

37. Holm L, Sander C. Dali: a network tool for protein structure comparison. Trends Biochem Sci 1990;20:478-480.

38. Kraulis PJ. MOLSCRIPT: a program to produce both detailed and schematic plots of protein structures. J Appl Crystallogr 1991;24: 946-950.

39. Esnouf RM. Further additions to MolScript version 1.4, including reading and contouring of electron-density maps. Acta Crystallogr D 1999;55:938-940. 